\title{
A SIMPLE PROOF THAT THE CONCORDANCE GROUP OF ALGEBRAICALLY SLICE KNOTS IS INFINITELY GENERATED ${ }^{1}$
}

\author{
BOJU JIANG (PO-CHU CHIANG)
}

\begin{abstract}
A simple proof of the result stated in the title is obtained by making the Casson-Gordon invariant additive.
\end{abstract}

A. J. Casson and C. McA. Gordon proved in [1], [2] that there are algebraically slice knots which are not slice knots. In other words, the concordance group $\mathbb{Q}$ of algebraically slice knots is nontrivial. A natural question: Is $\mathbb{Q}$ infinitely generated? The author learned from Wu-chung Hsiang that Casson had obtained the affirmative answer to this question for some time; however, Casson has not published his proof to date. The purpose of this short note is to present a simple proof, which would possibly be different from Casson's. The main point in our argument is the observation that we can make the Casson-Gordon invariant additive by slightly generalizing its definition, thereby making it possible to detect linear independence in $\mathcal{Q}$. We shall use the language and notation of [2].

1. Additivity of Casson-Gordon invariant for 3-manifolds. In [2], Casson and Gordon defined, for a closed oriented 3-manifold $M$ and an epimorphism $\phi$ : $H_{1}(M) \rightarrow \mathbf{Z}_{m}$, the invariant $\sigma_{r}(M, \phi), 0<r<m$. The definition goes as follows. Suppose $\tilde{M} \rightarrow M$ is the $m$-fold cyclic covering induced by $\phi$. Pick up an $m$-fold cyclic branched covering of 4-manifolds $\tilde{W} \rightarrow W$, branched over a surface $F \subset$ int $W$, such that $\partial(\tilde{W} \rightarrow W)=(\tilde{M} \rightarrow M)$. (The existence of such $(W, F)$ follows from Lemma 2.2 of [2].) Then define

$$
\sigma_{r}(M, \phi)=\operatorname{sign} W-\varepsilon_{r}(\tilde{W})-\frac{2[F]^{2} r(m-r)}{m^{2}} .
$$

For our purpose, we have to deal with arbitrary homomorphism $\phi$. For simplicity, we shall restrict ourselves to the case $m=p$, a prime, so that a homomorphism $H_{1}(M) \rightarrow \mathbf{Z}_{m}$ is either epimorphic or trivial.

Defintion. Let $\phi: H_{1}(M) \rightarrow Z_{p}$ be a homomorphism, where $M$ is an oriented closed 3-manifold, $p$ a prime. Define $\sigma_{r}(M, \phi)$ as above if $\phi$ is epimorphic, and define $\sigma_{r}(M, \phi)=0$ if $\phi$ is trivial, for $0<r<p$.

This invariant is additive in the following sense. Let $M^{\prime}, M^{\prime \prime}$ be two closed

Received by the editors June 30, 1980.

1980 Mathematics Subject Classification. Primary 55A25; Secondary 57D90.

${ }^{1}$ This work was done in Fall, 1979, while the author was visiting the Institute for Advanced Study, Princeton, New Jersey, and partially supported by an NSF grant. The author is grateful to Professor W. C. Hsiang for helpful conversations. 
oriented 3-manifolds. We know $H_{1}\left(M^{\prime} \# M^{\prime \prime}\right)=H_{1}\left(M^{\prime}\right) \oplus H_{1}\left(M^{\prime \prime}\right)$, so that every pair of homomorphisms $\phi^{\prime}: H_{1}\left(M^{\prime}\right) \rightarrow \mathbf{Z}_{p}, \phi^{\prime \prime}: H_{1}\left(M^{\prime \prime}\right) \rightarrow \mathbf{Z}_{p}$ determines uniquely a homomorphism $\phi=\phi^{\prime} \oplus \phi^{\prime \prime}: H_{1}\left(M^{\prime} \# M^{\prime \prime}\right) \rightarrow Z_{p}$, and vice versa.

LEMMA 1. $\sigma_{r}\left(M^{\prime} \# M^{\prime \prime}, \phi^{\prime} \oplus \phi^{\prime \prime}\right)=\sigma_{r}\left(M^{\prime}, \phi^{\prime}\right)+\sigma_{r}\left(M^{\prime \prime}, \phi^{\prime \prime}\right), 0<r<p$.

Proof. Case 1. Both of $\phi^{\prime}, \phi^{\prime \prime}$ are epimorphic. Let $\left(W^{\prime}, F^{\prime}\right)$ be the data needed for defining $\sigma_{r}\left(M^{\prime}, \phi^{\prime}\right)$, and $\left(W^{\prime \prime}, F^{\prime \prime}\right)$ be those for $\sigma_{r}\left(M^{\prime \prime}, \phi^{\prime \prime}\right)$. Take $(W, M)=$ $\left(W^{\prime}, M^{\prime}\right) \#\left(W^{\prime \prime}, M^{\prime \prime}\right)$ and $F=F^{\prime} \cup F^{\prime \prime}$. Then we may use $(W, F)$ for defining $\sigma_{r}\left(M^{\prime} \# M^{\prime \prime}, \phi^{\prime} \oplus \phi^{\prime \prime}\right)$. Now $W$ is obtained by pasting $W^{\prime}$ and $W^{\prime \prime}$ together along a 3-disk, and $\tilde{W}$ is obtained by pasting $\tilde{W}^{\prime}$ and $\tilde{W}^{\prime \prime}$ together along $p$ 3-disks, neither of which intersects the branching set $F$. By the Mayer-Vietoris sequence, we see that the intersection form on $H_{2}(W)$ is the orthogonal sum of those on $H_{2}\left(W^{\prime}\right)$ and $H_{2}\left(W^{\prime \prime}\right)$ (hence sign $W=\operatorname{sign} W^{\prime}+\operatorname{sign} W^{\prime \prime}$ and $[F]^{2}=\left[F^{\prime}\right]^{2}+\left[F^{\prime \prime}\right]^{2}$ ), and the intersection form on $H_{2}(\tilde{W})$ is the $\mathbf{Z}_{p}$-equivariant orthogonal sum of $H_{2}\left(\tilde{W}^{\prime}\right)$ and $H_{2}\left(\tilde{W}^{\prime \prime}\right)$ (hence $\left.\varepsilon_{r}(\tilde{W})=\varepsilon_{r}\left(\tilde{W}^{\prime}\right)+\varepsilon_{r}\left(\tilde{W}^{\prime \prime}\right)\right)$. Therefore

$$
\sigma_{r}\left(M^{\prime} \# M^{\prime \prime}, \phi^{\prime} \oplus \phi^{\prime \prime}\right)=\sigma_{r}\left(M^{\prime}, \phi^{\prime}\right)+\sigma_{r}\left(M^{\prime \prime}, \phi^{\prime \prime}\right), \quad 0<r<p .
$$

Case 2. $\phi^{\prime}$ is epimorphic but $\phi^{\prime \prime}$ is trivial. Let $\left(W^{\prime}, F^{\prime}\right)$ be the data needed for defining $\sigma_{r}\left(M^{\prime}, \phi^{\prime}\right)$. Take any $W^{\prime \prime}$ such that $\partial W^{\prime \prime}=M^{\prime \prime}$ and $H_{1}\left(W^{\prime \prime}\right)=0$. Take $(W, M)=\left(W^{\prime}, M^{\prime}\right) \#\left(W^{\prime \prime}, M^{\prime \prime}\right)$ and $F=F^{\prime}$. Then we may use $(W, F)$ for defining $\sigma_{r}\left(M^{\prime} \# M^{\prime \prime}, \phi^{\prime} \oplus \phi^{\prime \prime}\right)$. The same argument as in Case 1 still works if we take $F^{\prime \prime}$ to be empty and take $\tilde{W}^{\prime \prime}$ to be the disjoint union of $p$ copies of $W^{\prime \prime}$, with the obvious $\mathbf{Z}_{p}$ action by cyclic permutation. So we get sign $W=\operatorname{sign} W^{\prime}+$ sign $W^{\prime \prime}, \varepsilon_{r}(\tilde{W})=\varepsilon_{r}\left(\tilde{W}^{\prime}\right)+\varepsilon_{r}\left(\tilde{W}^{\prime \prime}\right),[F]^{2}=\left[F^{\prime}\right]^{2}$. But for this $\mathbf{Z}_{p}$-action on $\tilde{W}^{\prime \prime}$ it is easily seen that $\varepsilon_{r}\left(\tilde{W}^{\prime \prime}\right)=\operatorname{sign} W^{\prime \prime}$ for all $0<r<p$. Therefore

$$
\sigma_{r}\left(M^{\prime} \# M^{\prime \prime}, \phi \oplus \phi^{\prime \prime}\right)=\sigma_{r}\left(M^{\prime}, \phi^{\prime}\right)=\sigma_{r}\left(M^{\prime}, \phi^{\prime}\right)+\sigma_{r}\left(M^{\prime \prime}, \phi^{\prime \prime}\right), \quad 0<r<p .
$$

Case 3. Both of $\phi^{\prime}, \phi^{\prime \prime}$ are trivial. This case is trivial.

2. Additivity of Casson-Gordon invariant for knots. Let $K$ be a knot in $S^{3}, M_{n}(K)$ be the $2^{n}$-fold branched covering of $\left(S^{3}, K\right), \phi: H_{1}\left(M_{1}(K)\right) \rightarrow \mathrm{Z}_{m}$ be a homomorphism. By composition with the surjection induced by branched covering projection $M_{n}(K) \rightarrow M_{1}(K), \phi$ determines $\phi_{n}: H_{1}\left(M_{n}(K)\right) \rightarrow Z_{m}$. The Casson-Gordon invariant for $(K, \phi)$ is $\sigma_{r}\left(M_{n}(K), \phi_{n}\right), 0<r<m$. It was originally defined in [2] for epimorphic $\phi$, but now it also makes sense for arbitrary $\phi$ when $m=p$.

This invariant is additive in the following sense. Let $K^{\prime}, K^{\prime \prime}$ be two oriented knots in $S^{3}$. Then $M_{n}\left(K^{\prime} \# K^{\prime \prime}\right)=M_{n}\left(K^{\prime}\right) \# M_{n}\left(K^{\prime \prime}\right)$, so that every pair of homomorphisms $\phi^{\prime}: M_{1}\left(K^{\prime}\right) \rightarrow \mathbf{Z}_{p}$ and $\phi^{\prime \prime}: M_{1}\left(K^{\prime \prime}\right) \rightarrow \mathbf{Z}_{p}$ determines a unique $\phi=\phi^{\prime} \oplus \phi^{\prime \prime}: H_{1}\left(M_{1}\left(K^{\prime} \# K^{\prime \prime}\right)\right) \rightarrow Z_{p}$, and $\phi_{n}=\phi_{n}^{\prime} \oplus \phi_{n}^{\prime \prime}$. Now, a direct consequence of Lemma 1 is

LEMMA 2. $\sigma_{r}\left(M_{n}\left(K^{\prime} \# K^{\prime \prime}\right), \phi_{n}^{\prime} \oplus \phi_{n}^{\prime \prime}\right)=\sigma_{r}\left(M_{n}\left(K^{\prime}\right), \phi_{n}^{\prime}\right)+\sigma_{r}\left(M_{n}\left(K^{\prime \prime}\right), \phi_{n}^{\prime \prime}\right)$, for $0<$ $r<p$.

3. Doubled knots of the trivial knot. Let us quote from [2] some results about doubled knots. Let $K_{k}$ be the $k$-twisted double of the unknot, as depicted on [2,p. 46]. $K_{k}$ is known to be algebraically slice iff $4 k+1=l^{2}$ for some integer $l$. Let us 
rewrite $K_{k}$ as $K^{(l)}$ if $4 k+1=l^{2}$. Then $K^{(l)}, l$ taking odd values, are algebraically slice knots and represent elements of the concordance group $\mathcal{Q} . K^{(l)}$ is a slice knot iff $l=1$ or 3 . The computation in $[2, \S 5]$ can be summarized as

LEMMA 3. Let $\bar{\mu}_{1}$ be the generator of $H_{1}\left(M_{1}\left(K^{(l)}\right)\right)\left(\cong \mathbf{Z}_{l^{2}}\right)$ specified on $[2, p .48]$. Suppose an epimorphism $\phi: H_{1}\left(M_{1}\left(K^{(l)}\right)\right) \rightarrow \mathbf{Z}_{m}$ sends $\bar{\mu}_{1}$ to $q \in \mathbf{Z}_{m}$. Then

$$
\begin{aligned}
\lim _{n \rightarrow \infty} \frac{1}{2^{n}} \sigma_{r}\left(M_{n}\left(K^{(l)}\right), \phi_{n}\right)=2\left\{1+\left[\frac{\left(l^{2}-1\right) r^{\prime}}{2 m}\right]-r^{\prime}\left(m-r^{\prime}\right)\left(\frac{l}{m}\right)^{2}\right\}, & \\
& \text { for } 0<r<m,
\end{aligned}
$$

where $0<r^{\prime} \leqslant(m-1) / 2$ satisfies $r^{\prime} \equiv \pm q r(\bmod m)$. If $l>5$, the right-hand side is always negative.

In fact, the computation in [2] is carried out for $q=1$. But we can use the following general fact which can be easily proved by means of Novikov additivity: For a closed 3-manifold $M$ and two epimorphisms $\phi, \phi^{\prime}: H_{1}(M) \rightarrow \mathbf{Z}_{m}$, related by $\phi=q \phi^{\prime}$ where $q$ is coprime to $m$, we have $\sigma_{r}(M, \phi)=\sigma_{r}\left(M, \phi^{\prime}\right), 0<r<m$, where $r^{\prime} \equiv q r(\bmod m)$ and $0<r^{\prime}<m$.

\section{Infinite-generatedness of $\mathbb{Q}$.}

THEOREM. Let $P$ be the set of prime numbers $\geqslant 5$. Then, the set $\left\{K^{(p)}\right\}_{p \in P}$ is linearly independent in $\mathbb{Q}$.

For a proof, let us consider a knot

$$
K=k_{1} K^{\left(p_{1}\right)} \# \cdots \# k_{t} K^{\left(p_{t}\right)} \text {, }
$$

where $p_{1}, \ldots, p_{t} \in P, p_{i} \neq p_{j}$ for $i \neq j, k_{1}, \ldots, k_{t}$ are nonzero integers. We want to prove that $K$ is not slice. But in view of Theorem 4.1 of [2], it suffices to prove the following.

LEMMA 4. (1) For any subgroup $G$ of $H_{1}\left(M_{1}(K)\right)$ with $|G|^{2}=\left|H_{1}\left(M_{1}(K)\right)\right|$, there exists an epimorphism $\phi: H_{1}\left(M_{1}(K)\right) \rightarrow \mathbf{Z}_{p_{1}}$ satisfying $\phi(G)=0$.

(2) For any epimorphism $\phi: H_{1}\left(M_{1}(K)\right) \rightarrow \mathbf{Z}_{p_{1}}$,

$$
\begin{aligned}
\lim _{n \rightarrow \infty} \frac{1}{2^{n}} \sigma_{r}\left(M_{n}(K), \phi_{n}\right) & <0 & \text { if } k_{1}>0, \\
& >0 & \text { if } k_{1}<0 .
\end{aligned}
$$

Proof. (1) The factor group $H_{1}\left(M_{1}(K)\right) / G$ has order $|G|=p_{1}^{\left|k_{1}\right|} \cdots p_{t}^{\left|k_{l}\right|}$, hence it has $Z_{p_{1}}$ as a factor group. So there exists an epimorphism $\phi: H_{1}\left(M_{1}(K)\right) \rightarrow Z_{p_{1}}$.

(2) For short, let us write $M_{n}$ for $M_{n}(K), k_{i} M_{n}^{(i)}$ for $M_{n}\left(k_{i} K^{\left(p_{i}\right)}\right), K^{(i, j)}$ for the $j$ th copy of $\pm K^{\left(p_{i}\right)}$ in $k_{i} K^{\left(p_{i}\right)}, M_{n}^{(i, j)}$ for $M_{n}\left(K^{(i, j)}\right), 1 \leqslant j \leqslant\left|k_{i}\right|, 1 \leqslant i \leqslant t$. Then

$$
\begin{aligned}
M_{n} & =k_{1} M_{n}^{(1)} \# \cdots \# k_{t} M_{n}^{(t)}, \\
H_{1}\left(M_{1}\right) & =H_{1}\left(k_{1} M_{n}^{(1)}\right) \oplus \cdots \oplus H_{1}\left(k_{t} M_{n}^{(t)}\right) \\
& \cong\left(\mathbf{Z}_{p_{1}^{2}}\right)^{\left|k_{1}\right|} \oplus \cdots \oplus\left(\mathbf{Z}_{p_{t}^{2}}\right)^{\left|k_{t}\right|} .
\end{aligned}
$$


Recall that $-K$ means the mirror image of $K$, changing the sign of a knot also changes the sign of its Casson-Gordon invariant. Hence we only have to consider the $k_{1}>0$ case.

Write $\phi^{(i)}=\phi\left|H_{1}\left(k_{i} M_{1}^{(i)}\right), \phi^{(i, j)}=\phi\right| H_{1}\left(M^{(i, j)}\right)$. Then, for $i>1, \phi^{(i)}$ is trivial because $p_{i}$ is coprime to $p_{1}$. By Lemma 2 ,

$$
\sigma_{r}\left(M_{n}, \phi_{n}\right)=\sigma_{r}\left(k_{1} M_{n}^{(1)}, \phi_{n}^{(1)}\right)=\sum_{j=1}^{k_{1}} \sigma_{r}\left(M_{n}^{(1, j)}, \phi_{n}^{(1, j)}\right) .
$$

But $\phi$ is epimorphic, so that at least one of $\phi^{(1, j)}, 1<j<k_{1}$, will be epimorphic. The conclusion of the lemma then follows from Lemma 3.

The proof of the Theorem is now complete.

\section{REFERENCES}

1. A. J. Casson and C. McA. Gordon, Cobordism of classical knots, Mimeographed notes, Orsay, France, 1975.

2. __ On slice knots in dimension three, Proc. Sympos. Pure Math., vol 32, Amer. Math. Soc., Providence, R.I., pp. 39-53.

School of Mathematics, Institute for Advanced Study, Princeton, New Jersey 08540

Current address: Department of Mathematics, Peking University, Peking, China 